\title{
Limit Laws for Exponential Families
}

\author{
A.A. Balkema \\ Department of Mathemetics \\ University of Amsterdam \\ Pl. Muidergracht 24 \\ NL-1018TV Amsterdam, Holland \\ guus@wins.uva.nl
}

\author{
C. Klüppelberg* \\ Department of Mathematics \\ Technical University \\ D-80290 Munich, Germany \\ cklu@mathematik.tu-muenchen.de
}

\author{
S.I. Resnick ${ }^{\dagger}$ \\ Operations Research and Industrial Engineering \\ Rhodes Hall \\ Cornell University \\ Ithaca, New York 14853-7501 USA \\ sid@orie.cornell.edu
}

\begin{abstract}
For a real random variable $X$ with distribution function $F$ define

$$
\Lambda:=\left\{\lambda \in \mathbb{R}: K(\lambda):=E e^{\lambda X}<\infty\right\} .
$$

The distribution $F$ generates a natural exponential family of distribution functions $\left\{F_{\lambda}, \lambda \in \Lambda\right\}$, where

$$
d F_{\lambda}(x):=e^{\lambda x} d F(x) / K(\lambda) \quad \lambda \in \Lambda .
$$

We study the asymptotic behaviour of the distribution functions $F_{\lambda}$ as $\lambda$ increases to $\lambda_{\infty}:=\sup \Lambda$. If $\lambda_{\infty}=\infty$ then $F_{\lambda} \downarrow 0$ pointwise on $\{F<1\}$. It may still be possible to obtain a non-degenerate weak limit law $G(y)=\lim F_{\lambda}\left(a_{\lambda} y+b_{\lambda}\right)$ by choosing suitable scaling and centering constants $a_{\lambda}>0$ and $b_{\lambda}$ and in this case either $G$ is a Gaussian distribution or $G$ has a finite lower endpoint $y_{0}=\inf \{G>0\}$ and $G\left(y-y_{0}\right)$ is a gamma distribution. Similarly if $\lambda_{\infty}$ is finite and does not belong to $\Lambda$ then $G$ is a Gaussian distribution or $G$ has a finite upper endpoint $y_{\infty}$ and $1-G\left(y_{\infty}-y\right)$ is a gamma distribution. The situation for sequences $\lambda_{n} \uparrow \lambda_{\infty}$ is entirely different: Any distribution function may occur as the weak limit of a sequence $F_{\lambda_{n}}\left(a_{n} x+b_{n}\right)$.
\end{abstract}

AMS Subject Classifications: Primary 60F05; secondary 60E05

Key words: affine transformation, asymptotic normality, convergence of types, cumulant generating function, exponential family, Esscher transform, gamma distribution, Gaussian tail, limit law, normal distribution, moment generating function, power norming, semistable, stochastically compact, universal distributions.

\footnotetext{
*Author for correspondence.

${ }^{\dagger}$ Sidney Resnick was partially supported by NSF Grant DMS-97-04982 at Cornell University.
} 


\section{Introduction}

Suppose $X$ is a real random variable with df $F$. Let

$$
\Lambda=\left\{\lambda \in \mathbb{R} \mid K(\lambda):=E e^{\lambda X}<\infty\right\}
$$

be the set where the moment generating function (mgf) $K(\lambda)$ of $X$ is finite. The set $\Lambda$ is a connected subset of $\mathbb{R}$ which contains the origin and on which the mgf $\lambda \mapsto K(\lambda)$ is continuous and strictly positive. Associated with $F$ is the natural exponential family $\left\{F_{\lambda}, \lambda \in \Lambda\right\}$ where

$$
d F_{\lambda}(x):=e^{\lambda x} d F(x) / K(\lambda) \quad \lambda \in \Lambda
$$

For convenience, we let $X_{\lambda}$ be a random variable with distribution $F_{\lambda}$. We study the asymptotic behaviour of the df's $F_{\lambda}$ for $\lambda \rightarrow \lambda_{\infty}:=\sup \Lambda$. Note that $\lambda_{\infty} \geq 0$. We assume $\lambda_{\infty}>0$.

If $\lambda_{\infty} \in \Lambda$ then $F_{\lambda} \downarrow F_{\lambda_{\infty}}$ pointwise; see Corollary 2.1. If $\lambda_{\infty} \notin \Lambda$, then $F_{\lambda} \downarrow 1_{\left[x_{\infty}, \infty\right)}$ where $x_{\infty}=\sup \{F<1\}$ is the upper endpoint of the $\mathrm{df} F$; see Proposition 2.3. In the latter case, the types in the exponential family $\left\{F_{\lambda}, \lambda \in \Lambda\right\}$, may have a limit law for $\lambda \rightarrow \lambda_{\infty}$. This means that it may sometimes be possible to normalize the variables $X_{\lambda}$ of the exponential family by translation and positive scaling so that for some nonconstant random variable $Y$,

$$
A_{\lambda} X_{\lambda}:=\frac{X_{\lambda}-b_{\lambda}}{a_{\lambda}} \stackrel{\mathrm{d}}{\rightarrow} Y, \quad \lambda \rightarrow \lambda_{\infty} .
$$

Here $\stackrel{\mathrm{d}}{\rightarrow}$ denotes convergence in distribution.

This paper determines the possible non-degenerate limit laws in (1.3). Our main result, Theorem 3.3, states that if there is a non-constant limit variable $Y$ in (1.3), then one can choose the centering $b_{\lambda}$ and scaling $a_{\lambda}$ so that $Y$ is a standard normal variable, or so that $Y$ or $-Y$ has a gamma distribution. In a subsequent publication, we shall describe the domains of attraction of the limit laws.

This paper is partially motivated by Balkema, Klüppelberg and Resnick [1993], where it was found that asymptotic normality of $F_{\lambda}$ has useful implications for the study of sums of independent rv's. A class of thin tailed densities was identified which is closed under convolution This closure property is dependent on the fact that each density of the family had an associated exponential family which is asymptotically normal. Rootzen [1987] and 
Davis and Resnick [1991] use related ideas for applications to extremes of moving averages. Feigin and Yashchin [1983] and Balkema, Klüppelberg and Stadtmüller [1995] give Tauberian results based on the asymptotic normality of exponential families. If asymptotic normality of exponential families was useful for such things as convolution closure problems and Tauberian theory, we wondered what other weak limits could arise when converging to the boundary of $\Lambda$ and what applications were possible when convergence was to a nonnormal weak limit. The present paper is a first step in the exploration of applications of nonnormal limits.

The importance of exponential families in statistics and for asymptotics in probability theory can hardly be overestimated. In analysis exponential families occur as Esscher transforms and are used in Laplace's principle and for saddlepoint approximations. A survey of their use in statistics is Barndorff-Nielsen [1978], Barndorff-Nielsen and Cox [1994] or Brown [1986]. For connections with saddlepoint approximations, see Barndorff-Nielsen and Klüppelberg [1997] and Jensen [1995]. Limit behaviour of $F_{\lambda}$ is of mathematical interest and moreover the exponential family offers an effective way to investigate the asymptotic behaviour of the mgf $K$ and the cumulant generating function (cgf) $\kappa=\log K$.

Convergence in (1.3) depends on the behaviour of the $\operatorname{cgf} \kappa$ at $\lambda_{\infty}$. The behaviour of the analytic function $\kappa$ at a fixed point $\lambda_{0}<\lambda_{\infty}$ is well known:

$$
n \kappa\left(\lambda_{0}+\frac{t}{\sqrt{n}}\right)-n \kappa\left(\lambda_{0}\right)-\mu t \sqrt{n} \rightarrow \frac{\sigma^{2} t^{2}}{2} \quad n \rightarrow \infty .
$$

Here $\mu=\mu_{\lambda_{0}}=\kappa^{\prime}\left(\lambda_{0}\right)$ is the expectation of $X_{\lambda_{0}}$ and $\sigma^{2}=\sigma_{\lambda_{0}}^{2}=\kappa^{\prime \prime}\left(\lambda_{0}\right)$ the variance. Relation (1.4) is the formula for the second derivative of $\kappa$ at $\lambda_{0}$. It also is the Central Limit Theorem for sums of independent observations from the df $F_{\lambda_{0}}$ since $n\left(\kappa\left(\lambda_{0}+\xi\right)-\kappa\left(\lambda_{0}\right)\right)$ is the cgf of the df $F_{\lambda_{0}}^{* n}$. Teicher [1984] has investigated relation (1.4) for a sequence $\lambda_{n} \rightarrow \infty$, extending work of Feller [1969] on large deviations. More recently Broniatowski and Mason [1994] have looked at very large deviations. There the behaviour of the mgf for $\lambda \rightarrow \lambda_{\infty}$ plays a decisive role.

To understand the behaviour of the $\operatorname{cgf} \kappa$ for $\lambda \rightarrow \lambda_{\infty}$ assume existence of the following limit:

$$
\kappa_{\lambda}^{*}(t):=\kappa(\lambda+t / \sigma)-\kappa(\lambda)-\mu t / \sigma \rightarrow \eta(t) \quad \lambda \rightarrow \lambda_{\infty} .
$$

The right hand side of (1.5) describes the convex function $\kappa$ around the point $\lambda$ normalized so as to have a horizontal tangent at $t=0$ and curvature 1 . It is not surprising that the 
parabola $\eta(t)=t^{2} / 2$ occurs as a limit - corresponding to the normal law for the limit variable $Y$ in (1.3). The second limit function, the logarithm, corresponds to two families of gamma distributions. In Theorem 3.4 we prove that weak convergence (1.3) entails convergence of the cgf's. Hence we may use the first two moments of $X_{\lambda}$ to normalize, thus obtaining the limit relation (1.5).

Statistical applications have motivated interest in exponential families closed under certain transformation groups. Lehmann [1983] mentions exponential location families. Casalis [1991] classifies natural exponential families on $\mathbb{R}^{d}$ which are invariant under certain groups of affine transformations and Bar-Lev and Casalis [1994, 1998] describe exponential families $G_{\gamma}, \gamma \in \Gamma$, on $\mathbb{R}$ which are invariant under certain groups of affine transformations. For each $\gamma \in \Gamma$ there exist an $a>0$ and $b \in \mathbb{R}$ so that $G_{\gamma}(x)=G(a x+b)$.

The paper is organized as follows: In Section 2 we first prove certain continuity results. From these we derive a stability property for the limit variable $Y$ which allows us to obtain in Section 3 the possible limit distributions, the normal and gamma distributions. Section 4 comments briefly on limit relation (1.3) when convergence is only along sequences $\lambda_{n} \uparrow \lambda_{\infty}$ which makes the situation complex since then the cgf's need not converge. Example 4.6 shows that the Cauchy distribution may occur as weak limit and Theorem 4.8 shows that the behaviour of the convex function $\kappa$ may be quite bizarre.

In a following paper we shall describe domains of attraction and give an application to saddlepoint approximations.

This paper treats the asymptotic behaviour of the exponential family in the neighbourhood of the upper endpoint of $\Lambda$. The transformation $X^{\prime}=-X$ allows us to translate these results into statements about the asymptotic behaviour in the neighbourhood of the lower endpoint, $\inf \Lambda$. If $Y$ is a limit variable for the exponential family generated by $X$ in the upper endpoint then $-Y$ is a limit variable for the exponential family generated by $-X$ in the lower endpoint.

Obviously the multivariate case is the really interesting situation. The setting there is simple: The cgf of a random vector is a convex function defined (finite) on a convex subset $\Lambda \subset \mathbb{R}^{d}$. For simplicity assume $\Lambda$ is open. The cgf is analytic. What is its behaviour as one approaches the boundary? Normalize the cgf for $\lambda_{0} \in \Lambda$ so that the tangent hyperplane in $\lambda_{0}$ is horizontal and the second derivative is the standard inner product. The associated random vector $X_{\lambda}^{*}$ has zero expectation vector and the identity matrix as covariance. What 
happens to the distribution of $X_{\lambda}^{*}$ as $\lambda$ approaches a point on the boundary of $\Lambda$ or tends to infinity? Do there exist non-degenerate limit laws? Do the mgf's of $X_{\lambda}^{*}$ converge? Is it possible that $X_{\lambda}^{*}$ does not converge in distribution but that $X_{\lambda}$ does converge for some other normalization?

This paper will answer some of these questions in the univariate case.

\section{Stability of the limit laws}

Random variables arising from a limit procedure frequently satisfy a stability condition. For the df $G$ of the limit $Y$ of the exponential family in (1.3), the stability relation takes on the form

$$
G_{\gamma}(x)=G(a x+b) \quad a>0, b \in \mathbb{R} .
$$

Indeed $G$ satisfies a large number of such relations. The random variables $Y_{\gamma}$ in the exponential family of the limit variable $Y$ all are of the same type! The essential step in establishing this stability for the limit variable is Proposition 2.12.

We start by studying the behaviour of $\left\{F_{\lambda}\right\}$ as $\lambda \uparrow \lambda_{\infty}=\sup \Lambda$ without using any normalization. We then consider the following question: Suppose a sequence of df's $F_{n}$ converges weakly to a non-degenerate df $F$. Let $G_{n}=\left(F_{n}\right)_{\lambda_{n}}$ be a df in the exponential family of $F_{n}$ and suppose $G_{n} \rightarrow G$ weakly. What is the relation between the limit distributions $F$ and $G$ ? We answer this question in Theorem 2.7. In the second part of this section we consider weak limit behaviour under positive affine transformations and consider $F_{\lambda}\left(a_{\lambda} x+b_{\lambda}\right)$ for $\lambda \rightarrow \lambda_{\infty}$. The norming constants $a_{\lambda}>0$ and $b_{\lambda}$ may be chosen to vary continuously with $\lambda$. The limit distribution will depend on the normalization. By Khintchine's convergence of types theorem different non-degenerate limit distributions will belong to the same type.

Proposition 2.1 For any fixed $x$ for which $0<F(x)<1$ the function $\lambda \mapsto F_{\lambda}(x)$ is strictly decreasing on $\Lambda$ and continuous. It is also true that $\lambda \mapsto F_{\lambda}(x-)$ is strictly decreasing and continuous on $\Lambda$.

Proof For monotonicity see Brown [1986, Cor. 2.22]. For continuity, if $\lambda \rightarrow \lambda_{0}$, convergence of

$$
\int 1_{(-\infty, x]} e^{\lambda u} d F(u)=K(\lambda) F_{\lambda}(x) \rightarrow K\left(\lambda_{0}\right) F_{\lambda_{0}}(x)
$$


follows by dominated convergence with dominating function $e^{\alpha u} \vee e^{\beta u}$ with $\alpha, \beta \in \Lambda$. The continuity of the mgf $K$ on $\Lambda$ gives $F_{\lambda}(x) \rightarrow F_{\lambda_{0}}(x)$.

Corollary 2.2 If $\lambda_{\infty}=\sup \Lambda \in \Lambda$, then $F_{\lambda} \downarrow F_{\lambda_{\infty}}$ for $\lambda \uparrow \lambda_{\infty}$.

The interesting case is when the upper endpoint $\lambda_{\infty}$ does not lie in $\Lambda$.

Proposition 2.3 Suppose $\lambda_{\infty} \notin \Lambda$. Let $x_{\infty}=\sup \{F<1\} \leq \infty$ denote the upper endpoint of the df F. Then $F_{\lambda} \downarrow 1_{\left[x_{\infty}, \infty\right)}$.

Proof If $\lambda_{\infty}$ is finite, then $x_{\infty}=\infty$ and $K(\lambda) \rightarrow \infty$ for $\lambda \rightarrow \lambda_{\infty}$. (Else $K\left(\lambda_{\infty}-0\right)<\infty$ and $\lambda_{\infty} \in \Lambda$ by Fatou's lemma.) Hence for any $x_{1}>0$

$$
F_{\lambda}\left(x_{1}\right)=\int_{\infty}^{x_{1}} e^{\lambda x} d F(x) / K(\lambda) \leq e^{\lambda_{\infty} x_{1}} / K(\lambda) \rightarrow 0 \quad \lambda \rightarrow \lambda_{\infty} .
$$

Now assume $\lambda_{\infty}=\infty$. If $F$ is degenerate the statement is obvious. Else choose $x_{1}<$ $x_{2}<x_{\infty}$ so that $F\left(x_{1}\right)>0$. Then $1-F\left(x_{2}\right)=p>0$, and

$$
\frac{1-F_{\lambda}\left(x_{2}\right)}{F_{\lambda}\left(x_{1}\right)}=\frac{\int_{\left(x_{2}, \infty\right)} e^{\lambda x} d F(x)}{\int_{\left(-\infty, x_{1}\right]} e^{\lambda x} d F(x)} \geq \frac{e^{\lambda x_{2}} p}{e^{\lambda x_{1}}} \rightarrow \infty \quad \lambda \rightarrow \infty .
$$

Since $1-F_{\lambda}\left(x_{2}\right) \leq 1$, we have $F_{\lambda}\left(x_{1}\right) \rightarrow 0$.

For convenience, we associate to each $\lambda \in \Lambda$ a $\mathrm{rv} X_{\lambda}$ with df $F_{\lambda}$. We shall write $X_{\lambda}=: E_{\lambda} X$ where $E_{\lambda}$ denotes the Esscher operator. The Esscher operators $E_{\lambda}$ satisfy the additive law

$$
E_{\mu} E_{\lambda}=E_{\lambda+\mu} \quad \lambda, \lambda+\mu \in \Lambda .
$$

Now suppose $X_{n}$ are rv's and $Y_{n}=E_{\gamma_{n}} X_{n}$ for some sequence $\gamma_{n}$. Let $X_{n}$ converge to $X_{0}$ in distribution and $Y_{n}$ to $Y_{0}$. Does it follow that $\gamma_{n} \rightarrow \gamma_{0}$ and $Y \stackrel{\text { d }}{=} E_{\gamma} X_{0}$ ?

Proposition 2.4 Suppose $X_{n} \stackrel{\mathrm{d}}{\rightarrow} X_{0}$ and $\gamma_{n} \rightarrow \gamma_{0}$. Let $X_{n}$ have $m g f K_{n}$ for $n \geq 0$. Assume that $K_{n}\left(\gamma_{n}\right)$ is finite for $n \geq 1$ and write $Y_{n}=E_{\gamma_{n}} X_{n}$.

(1) If $K_{n}\left(\gamma_{n}\right) \rightarrow K_{0}\left(\gamma_{0}\right)<\infty$, then $Y_{n} \stackrel{\mathrm{d}}{\rightarrow} Y_{0}$.

(2) If $Y_{n} \stackrel{\mathrm{d}}{\rightarrow} Y$ for some $r v Y$, then $Y=E_{\gamma_{0}} X_{0}$ and $K_{n}\left(\gamma_{n}\right) \rightarrow K_{0}\left(\gamma_{0}\right)<\infty$.

Proof Let $a_{n}=K_{n}\left(\gamma_{n}\right)$ and let $\pi_{n}$ be the distribution of $X_{n}$ for $n \geq 0$. Set $d \mu_{n}(x)=$ $e^{\gamma_{n} x} d \pi_{n}(x)$. Then $a_{n}=\mu_{n}(\mathbb{R})$ for $n \geq 0$ and $Y_{n}$ has distribution $\rho_{n}:=\mu_{n} / a_{n}$ for $n \geq 1$. 
Convergence $\int \varphi d \mu_{n} \rightarrow \int \varphi d \mu_{0}$ holds for continuous functions $\varphi$ with compact support. This means that $\mu_{n} \rightarrow \mu_{0}$ vaguely and hence $\mu_{0}(O) \leq \liminf \mu_{n}(O)$ for open sets $O$. For $O=\mathbb{R}$ this implies

$$
a_{0} \leq \liminf a_{n} .
$$

To prove (1), note if $a_{n} \rightarrow a_{0}<\infty$, then $\mu_{n} \rightarrow \mu_{0}$ weakly and this also holds for the normalized measures so that $Y_{n} \stackrel{\mathrm{d}}{\rightarrow} Y_{0}$.

For (2), suppose $\rho_{n} \rightarrow \rho$ weakly for some probability measure $\rho$. Write

$$
d \pi_{n}(x)=a_{n} e^{-\gamma_{n} x} d \rho_{n}(x)
$$

If $a_{n} \rightarrow \infty$ then $\rho_{n}(K) \rightarrow 0$ for any compact interval $K$, and hence $\rho_{n} \rightarrow 0$ vaguely. This contradicts the assumption that $\rho_{n} \rightarrow \rho$ weakly. Similarly $a_{n} \rightarrow 0$ implies $\pi_{n}(K) \rightarrow 0$ which contradicts the weak convergence $\pi_{n} \rightarrow \pi_{0}$. Hence the sequence $\left(a_{n}\right)$ is bounded. We claim that $a_{0}$ is the only limit point (which proves (2)). Any limit point " $a$ " satisfies $a_{0} \leq a$ from (2.2). On interchanging the role of $X_{n}$ and $Y_{n}$ we find $1 / a_{0} \leq 1 / a$. The two inequalities combined give $a=a_{0}$.

Example 2.5 The sequence $K_{n}\left(\gamma_{n}\right)$ in Proposition 2.4 may converge to a finite limit $a \neq K_{0}\left(\gamma_{0}\right)$.

Construction Take $\gamma_{n}=1$ for all $n$ and let $\rho_{n}$ have mass $1 / 2$ in the two points 0 and $x_{n}=n$. Then $X_{n} \stackrel{\mathrm{d}}{\rightarrow} X_{0} \equiv 0$ and $a_{n}=1 /\left(1 / 2+e^{-n} / 2\right) \rightarrow a=2>1=a_{0}$.

Example 2.6 It may happen that $X_{n} \stackrel{\mathrm{d}}{\rightarrow} X, Y_{n}=E_{\gamma_{n}} X_{n} \stackrel{\mathrm{d}}{\rightarrow} Y$ and $\gamma_{n} \rightarrow \infty$.

Construction Let $\mu$ be a finite measure which charges both $(-\infty, 0)$ and $(0, \infty)$. Let $\pi_{n}$ be the probability measure $c_{n}\left(1 \wedge e^{-n x}\right) d \mu(x)$ for $n \geq 0$. Take $\gamma_{n}=n$. The $\operatorname{rv} Y_{n}=E_{\gamma_{n}} X_{n}$ has distribution $d \rho_{n}(x)=b_{n}\left(e^{n x} \wedge 1\right) d \mu(x)$. It is clear that $X_{n}$ converges in distribution to a rv $X$ with probability distribution $d \pi=c 1_{(-\infty, 0]} d \mu$ and $Y_{n}$ to a $\operatorname{rv} Y$ with distribution $d \rho=b 1_{[0, \infty)} d \mu$.

We can now prove a kind of convergence of types theorem where "type" has to be interpreted as belonging to the same exponential family.

Theorem 2.7 Let $Y_{n}=E_{\gamma_{n}} X_{n}$ for $n \geq 1$ and $a_{n}=E e^{\gamma_{n} X_{n}}$. Suppose $X_{n} \stackrel{\mathrm{d}}{\rightarrow} X$ with $X$ non-constant, and $Y_{n} \stackrel{\mathrm{d}}{\rightarrow} Y$. 
If $\left(\gamma_{n}\right)$ is bounded, then $\gamma_{n} \rightarrow \gamma, a_{n} \rightarrow a=E e^{\gamma X}<\infty$ and $Y=E_{\gamma} X$.

If $\sup \gamma_{n}=\infty$ then $\gamma_{n} \rightarrow \infty$ and there exists a point $c \in \mathbb{R}$ so that $X \leq c \leq Y$ a.s.

If inf $\gamma_{n}=-\infty$ then $\gamma_{n} \rightarrow-\infty$ and there exists a point $c$ so that $Y \leq c \leq X$ a.s.

Proof First consider the case $\gamma_{n} \rightarrow \infty$. Suppose the distributions overlap: There exist $a<b$ so that $P\{X>b\}>0$ and $P\{Y<a\}>0$. Let $\delta$ denote the minimum of these two positive numbers. Then $P\left\{X_{n}>b\right\}$ and $P\left\{Y_{n}<a\right\}$ eventually exceed $\delta / 2$. Thus eventually

$$
\frac{\delta / 2}{1-\delta / 2} \leq \frac{P\left\{Y_{n}<a\right\}}{P\left\{Y_{n}>b\right\}} \leq \frac{e^{\gamma_{n} a} P\left\{X_{n}<a\right\}}{e^{\gamma_{n} b} P\left\{X_{n}>b\right\}} \leq e^{-\gamma_{n}(b-a)} \frac{1-\delta / 2}{\delta / 2}
$$

This contradicts the assumption that $\gamma_{n} \rightarrow \infty$.

The case $\gamma_{n} \rightarrow-\infty$ is treated in the same way.

There are three mutually exclusive alternatives: Either (i) $X \leq c \leq Y$, or (ii) $Y \leq c \leq$ $X$, or (iii) neither (i) nor (ii) hold. Hence the sequence $\gamma_{n}$ is bounded, or it diverges to $+\infty$ or it diverges to $-\infty$. If $\left(\gamma_{n}\right)$ is bounded, then by Proposition 2.4, $\gamma_{n}$ converges to some value $\gamma$ since the Esscher transforms $E_{\alpha} X$ and $E_{\beta} X$ are different for $\alpha \neq \beta$ if $X$ is not constant.

Now return to the exponential family $\left\{X_{\lambda}, \lambda \in \Lambda\right\}$ and assume that $\lambda_{\infty} \notin \Lambda$. To obtain a non-degenerate limit distribution for the variables $X_{\lambda}$ in the case $\lambda_{\infty} \notin \Lambda$, we have to normalize these variables, so assume (1.3) holds, $\left(X_{\lambda}-b_{\lambda}\right) / a_{\lambda} \stackrel{\mathrm{d}}{\rightarrow} Y$ for some non-constant random variable $Y$. By Proposition 2.1 the family $\left\{F_{\lambda}\right\}$ of $X_{\lambda}$ is weakly continuous in $\lambda$. This makes it possible to choose the coefficients $a_{\lambda}>0$ and $b_{\lambda} \in \mathbb{R}$ to be continuous on $\Lambda$.

Lemma 2.8 The constants $a_{\lambda}$ and $b_{\lambda}$ in (1.3) can be chosen to be continuous functions of $\lambda$ on the set $\Lambda$.

Proof Write $Y=\psi(U)$ with $\psi$ increasing and $U$ uniform $(0,1)$. One may take for $\psi$ the left-continuous inverse of the df of $Y$. Choose $p \in(0,1 / 2)$ so small that $\psi(p)<\psi(1-p)$. Set

$$
b:=\int_{p / 2}^{1-p / 2} \psi(u) d u \quad a:=\int_{1-p}^{1-p / 2} \psi(u) d u-\int_{p / 2}^{p} \psi(u) d u .
$$

Let $Y^{\prime}$ denote the normalized variable $(Y-b) / a$. Similarly write $X_{\lambda}=\varphi_{\lambda}(U)$ and define the smoothed median $b_{\lambda}$ and smoothed range $a_{\lambda}$ as above with $\varphi_{\lambda}$ replacing $\psi$. Then 
$a_{\lambda}>0$ eventually and convergence $A_{\lambda} X_{\lambda} \stackrel{\mathrm{d}}{\rightarrow} Y$ for some family of normalizations $A_{\lambda}$ implies convergence $\left(X_{\lambda}-b_{\lambda}\right) / a_{\lambda} \stackrel{\mathrm{d}}{\rightarrow} Y^{\prime}$. Weak continuity of $\lambda \mapsto \varphi_{\lambda}$ is equivalent to weak continuity of the exponential family $F_{\lambda}$ and implies continuity of the norming constants $a_{\lambda}$ and $b_{\lambda}$.

We will need the fact that Esscher operators react in a simple way with scaling and translation:

$$
E_{\lambda / a}(a X+b) \stackrel{d}{=} a E_{\lambda} X+b
$$

for $\lambda \in \Lambda, a>0$ and $b \in \mathbb{R}$. This follows since both sides of (2.3) have the same mgf $z \mapsto e^{b z} K(a z+\lambda) / K(\lambda)$.

We now discuss the stability property of the limit variable $Y$ in (1.3). Let $M(\gamma)=E e^{\gamma Y}$ be the mgf of $Y$ and $\left\{Y_{\gamma}, \gamma \in \Gamma\right\}$, the associated exponential family with $\Gamma=\{M<\infty\}$. We shall see below that there exist many pairs $(\gamma, C)$ with $\gamma \in \Gamma$ and $C$ in the group $\mathcal{G}$ of positive affine transformations $x \mapsto C(x)=(x-b) / a$ with $a>0$ and $b \in \mathbb{R}$ which satisfy the stability relation

$$
E_{\gamma} Y \stackrel{\mathrm{d}}{=} C Y \text {. }
$$

Example 2.9 The extended gamma family.

Construction The following variables satisfy (2.4) for all $\gamma$ for which the mgf of $Y$ is finite.

1) If $Y$ is $N\left(\mu, \sigma^{2}\right)$-distributed then $Y_{\gamma} \stackrel{\text { d }}{=} Y+\sigma^{2} \gamma$ for $\gamma \in \Gamma=\mathbb{R}$.

2) The standard exponential rv satisfies the relation $Y_{\gamma} \stackrel{\mathrm{d}}{=} Y /(1-\gamma)$ for $\gamma<1$. Similarly $Z=-Y$ satisfies the relation $Z_{\gamma} \stackrel{\mathrm{d}}{=} Z /(1+\gamma)$ for $\gamma>-1$.

3) More generally if $Y$ (or $-Z$ ) has a gamma density $x^{s-1} e^{-x} / \Gamma(s)$ on $(0, \infty)$ then $Y_{\gamma} \stackrel{\mathrm{d}}{=} Y /(1-\gamma)$ for $\gamma<1$ (and $Z_{\gamma} \stackrel{\mathrm{d}}{=} Z /(1+\gamma)$ for $\gamma>-1$ ).

These rv's generate exponential families whose df's are all of the same type.

Since the gamma distribution with shape parameter $s$ is asymptotically normal for $c=1 / s \rightarrow 0$ we have a continuous three parameter family of df's $H_{c}(a x+b), a>0, b$ and $c$ real. Here $H_{0}$ is the standard normal df, $H_{-c}(x)=1-H_{c}(-x)$ for $c>0$, and $H_{c}$ is the df of the normalized gamma variable $V_{c}=(Y-s) / \sqrt{s}$, with $c=1 / s$, where $Y$ has density $x^{s-1} e^{-x} / \Gamma(s)$ on $(0, \infty)$. 
Our main result states: This three parameter extended gamma family is the set of limit laws for exponential families, both for $\lambda \rightarrow \sup \Lambda$ and for $\lambda \rightarrow \inf \Lambda$.

Note the resemblance to extreme value limit theory where there also is a continuous three parameter family of limit distributions. See de Haan [1970, p. 104]. This resemblance is not due to some innate relation between extremes and exponential families, but results from the structure of the group $\mathcal{G}$ of positive affine transformations on $\mathbb{R}$. The group $\mathcal{G}$ has two kinds of elements: Translations, and multiplications with a given center. The normal distributions are stable for translations; the gamma distributions with a given endpoint are stable for multiplications having the endpoint as center. The extended gamma family reflects this structure.

We now want to show that the limit variable $Y$ in (1.3) has to satisfy a number of stability relations of the form (2.4).

With the positive affine transformation $A$ in $\mathcal{G}$, given by $A x=(x-b) / a$ we associate the point $(\log a, b)$ in the plane. It is then natural to set

$$
\|A\|:=\|(\log a, b)\|_{2}=\sqrt{(\log a)^{2}+b^{2}} .
$$

The function $\|\cdot\|$ is not a norm on the group $\mathcal{G}$, in particular $\left\|A^{-1}\right\| \neq\|A\|$, but it does describe the topology of $\mathcal{G}$ adequately for our purpose.

Proposition 2.10 Let $U_{\gamma}, \gamma \in \Gamma$, be the exponential family generated by the non-constant rv $U$. Suppose $C_{n} \in \mathcal{G}, \gamma_{n} \in \Gamma, C_{n} U_{\gamma_{n}} \stackrel{\mathrm{d}}{\rightarrow} Z$ with $Z$ non-constant, $\gamma_{n} \rightarrow \gamma>0$. Then

$$
\left\|C_{n}\right\| \rightarrow \infty \quad \text { iff } \quad \gamma \notin \Gamma
$$

Proof Suppose $\gamma \in \Gamma$. Then $E_{\gamma_{n}} U \stackrel{\mathrm{d}}{\rightarrow} E_{\gamma} Y$ by Proposition 2.1. The convergence of types theorem implies that $Z=C U_{\gamma}$ and $C_{n} \rightarrow C$ with $\|C\|<\infty$. For the converse assume that $\left(C_{n}\right)$ contains a convergent subsequence, say $C_{n} \rightarrow C$ as $n \rightarrow \infty$. Then $E_{\gamma_{n}} U \stackrel{\mathrm{d}}{\rightarrow} C^{-1} Z$. Proposition 2.4 implies $\gamma \in \Gamma$.

In order to characterise the possible limit distributions in (1.3), we need equation (2.4) to hold for a large collection of $\gamma$-values.

Lemma 2.11 Suppose (1.3) holds. For any $r>0$ and $\mu \in\left[0, \lambda_{\infty}\right)$ there exists $\lambda \in\left(\mu, \lambda_{\infty}\right)$ such that

$$
\left\|A_{\mu} A_{\lambda}^{-1}\right\| \vee(\lambda-\mu) a_{\mu}=r .
$$


Proof Write $Y^{\lambda}=A_{\lambda} X_{\lambda}$. (The upper index notation is used here to avoid confusion with the exponential family generated by the variable $Y$.) Fix $\mu \in \Lambda$. Use (2.3) and write

$$
A_{\mu} A_{\lambda}^{-1} Y^{\lambda} \stackrel{\mathrm{d}}{=} E_{(\lambda-\mu) a_{\mu}} Y^{\mu} \quad \lambda \in \Lambda \text {. }
$$

By assumption $Y^{\lambda} \stackrel{\mathrm{d}}{\rightarrow} Y$ for $\lambda \rightarrow \lambda_{\infty}$. Apply Proposition 2.10 with $E_{(\lambda-\mu) a_{\mu}} Y^{\mu}$ in the role of $U_{\gamma_{n}}$ and $\left(A_{\mu} A_{\lambda}^{-1}\right)^{-1}$ in the role of $C_{n}$ to conclude that $\left\|\left(A_{\mu} A_{\lambda}^{-1}\right)^{-1}\right\| \rightarrow \infty$ as $\lambda \rightarrow \lambda_{\infty}$. Check that $\left\|C_{n}\right\| \rightarrow \infty$ if $\left\|C_{n}^{-1}\right\| \rightarrow \infty$. By Lemma 2.8 the quantity $\left\|A_{\mu} A_{\lambda}^{-1}\right\|$ varies continuously from 0 to $\infty$ as $\lambda$ increases from $\mu$ to $\lambda_{\infty}$. So the leftmost term in (2.6) will equal $r$ before $\lambda$ reaches the value $\lambda_{\infty}$.

Fix $r>0$. Let $\mu_{n} \rightarrow \lambda_{\infty}$ and choose $\lambda_{n}>\mu_{n}$ as in the Lemma. Choose a subsequence $k_{1}<k_{2}<\cdots$ so that

$$
C_{k_{n}}:=A_{\mu_{k_{n}}} A_{\lambda_{k_{n}}}^{-1} \rightarrow C \in \mathcal{G}, \quad \gamma_{k_{n}}:=\left(\lambda_{k_{n}}-\mu_{k_{n}}\right) a_{\mu_{k_{n}}} \rightarrow \gamma
$$

This is possible since $\left\|C_{n}\right\|$ and $\gamma_{n}$ are bounded by $r$. Then $C_{k_{n}} Y^{\lambda_{k_{n}}} \stackrel{\mathrm{d}}{=} E_{\gamma_{k_{n}}} Y^{\mu_{k_{n}}}$ by (2.7). Theorem 2.7 gives $C Y \stackrel{\mathrm{d}}{=} Y_{\gamma}$ with $\|C\| \vee \gamma=r$ by continuity. This establishes the next result:

Proposition 2.12 If (1.3) holds and $Y$ is non-degenerate then for each $r>0$ there exists a constant $\gamma>0$ and a positive affine transformation $C$ with $\|C\| \vee \gamma=r$ such that (2.4) holds: $E_{\gamma} Y \stackrel{\mathrm{d}}{=} C Y$.

The question whether all distributions in the exponential family of the limit distribution are of the same type will be settled by algebraic arguments in the next section.

\section{Solutions of the stability equation}

The stability equation (2.4), $E_{\gamma} Y \stackrel{\mathrm{d}}{=} C Y$, allows us to determine the possible limit laws for the exponential family $X_{\lambda}$ for $\lambda \rightarrow \lambda_{\infty}$.

For statistical applications it is of importance to characterize exponential families which are invariant under a given group $\mathcal{H}$ of transformations. Lehmann [1983, p.35] observes that the normal distributions with fixed variance constitute an exponential family which also is a location family, and that the random variable $c \log Y$ with $Y$ gamma is the only other variable which generates such a family. This result is due to Dynkin in 1951. For the simpler case of natural exponential families Casalis [1991] in a very readable paper has 
solved the characterization problem when $\mathcal{H}$ is a group of translations on $\mathbb{R}^{d}$ and for some other classical groups of affine transformations on $\mathbb{R}^{d}$. Bar-Lev and Casalis [1994, 1998] solve the problem for the case when $\mathcal{H}$ is a subgroup of the group of affine transformations on $\mathbb{R}$. We are grateful to a referee of a previous version of this paper for pointing out these two references. We understand that full proofs of the statements in the second paper will be published shortly and restrict ourselves here to a short exposition of the results of this paper which are relevant to us.

If a natural exponential family is invariant under a group $\mathcal{H}$ of positive affine transformations, and the df's are non-degenerate then $\mathcal{H}$ is a closed commutative subgroup of $\mathcal{G}$. If $\mathcal{H}$ is a group of translations then the smallest interval which supports the distribution functions $G_{\xi}, \xi \in \Gamma$, of the invariant exponential family is the whole line. If $\mathcal{H}$ is a group of multiplications with center $q$ the smallest interval is the half line $(-\infty, q)$ or $(q, \infty)$. From these results one may conclude that the group $\mathcal{H}$ either is the discrete group $C^{k}$, $k \in \mathbb{Z}$, generated by a positive affine transformation $C \neq \mathrm{id}$, or the one dimensional group $C^{t}, t \in \mathbb{R}$. There are other closed subgroups of $\mathcal{G}$ but these do not enter the picture.

In the present paper we are concerned with the more elementary question of describing all df's $G$ which satisfy one or more stability relations of the form (2.1). Let $\mathcal{F}(\gamma, C)$ denote the set of all df's $G$ which satisfy the relation $G(a x+b)=G_{\gamma}(x)$ for $C x=(x-a) / b$.

Example 3.1 There exist rv's $V$ which satisfy the relation $V_{\gamma} \stackrel{\mathrm{d}}{=} V+\beta$ only if $\gamma$ and $\beta$ are integers.

Construction Let $V$ be the random integer with distribution

$$
P\{V=k\}=p_{k}=e^{-k^{2} / 2} / c \quad k \in \mathbb{Z}
$$

with $c$ a norming constant. The rv $V_{\xi}$ has distribution $P\left\{V_{\xi}=k\right\}=e^{-(k-\xi)^{2} / 2} / C(\xi)$. If $\gamma$ is an integer then $V_{\gamma} \stackrel{\mathrm{d}}{=} V+\gamma$, but if $\gamma$ is not an integer then $V$ and $V_{\gamma}$ are not of the same type.

We shall now show that for $G \in \mathcal{F}(\gamma, C)$ the exponential family $G_{\xi}, \xi \in \Gamma$, generated by $G$ is invariant under the discrete affine group $\mathcal{H}$ generated by $C$.

Proposition 3.2 Let $Y_{\xi}, \xi \in \Gamma$, be an exponential family. Suppose $\alpha \neq \beta$ lie in $\Gamma$ and 
$Y_{\beta} \stackrel{\mathrm{d}}{=} C Y_{\alpha}$ where $C x=(x-b) / a$ with $a>0$. Then for all integers $t$ and all $\xi \in \Gamma$

$$
C^{t} Y_{\xi} \stackrel{\mathrm{d}}{=} Y_{C_{*}^{t} \xi} \quad C_{*}^{t} \xi= \begin{cases}\xi+(\beta-\alpha) t & \text { if } a=1 \\ a^{t} \xi+\frac{a^{t}-1}{a-1}(\beta-a \alpha) & \text { if } a \neq 1 .\end{cases}
$$

If $Y$ belongs to the extended gamma family then these relations hold for all $t \in \mathbb{R}$. Moreover for each $\gamma_{0} \in \Gamma$ the map $t \mapsto \gamma_{t}=C_{*}^{t} \gamma_{0}$ is a strictly increasing function from $\mathbb{R}$ onto $\Gamma$. It satisfies the differential equation

$$
\ddot{\gamma}=r \dot{\gamma} \quad \gamma(0)=\gamma_{0}, \gamma(1)=a\left(\gamma_{0}-\alpha\right)+\beta, r=\log a .
$$

Proof Equation (3.2) is an application of the relation (2.3). First assume $\alpha \neq 1$. Write $\xi=\alpha+\gamma$. Then

$$
C Y_{\xi}=C Y_{\alpha+\gamma}=C E_{\gamma} Y_{\alpha} \stackrel{\mathrm{d}}{=} E_{a \gamma} C Y_{\alpha}=E_{a \gamma} Y_{\beta}=Y_{\beta+a \gamma}=Y_{C_{*} \xi}
$$

with

$$
C_{*} \xi=\beta+a(\xi-\alpha)=a(\xi-\pi)+\pi \quad \pi(1-a)=\beta-a \alpha .
$$

Hence $C^{t} Y_{\xi} \stackrel{\mathrm{d}}{=} Y_{C_{*}^{t} \xi}$ with $C_{*}^{t} \xi=a^{t} \xi+\left(1-a^{t}\right) \pi=a^{t} \xi+\left(a^{t}-1\right)(\beta-a \alpha) /(a-1)$.

The case $a=1$ is simpler.

The second part follows by a continuity argument (or direct inspection). Surjectivity of the map $\gamma$ results from Proposition 2.10.

Since the limit variable $Y$ in (1.3) satisfies a stability relations of the form (2.1) with $\|C\| \vee \gamma=r$ for each $r>0$ the exponential family is invariant under a continuous group $\mathcal{H}$. From the results of Bar-Lev and Casalis [1994, 1998] it follows that $Y$ has a Gaussian distribution if $\mathcal{H}$ is the group of translations and that there exists a real $d \neq 0$ so that $(Y-q) / d$ has a standard gamma distribution on $(0, \infty)$ if $\mathcal{H}$ is the group of all multiplications with center $q$. This gives:

Theorem 3.3 Let $F_{\lambda}, \lambda \in \Lambda$, be the exponential family (1.1). If $\lambda_{\infty}=\sup \Lambda$ does not belong to $\Lambda$ and if there exist constants $a_{\lambda}>0$ and $b_{\lambda} \in \mathbb{R}$ so that $F_{\lambda}\left(a_{\lambda} x+b_{\lambda}\right) \rightarrow G$ weakly for some non-degenerate df $G$ then $G$ belongs to the extended gamma family introduced in Example 2.9.

The exponential families of gamma distributions are generated by Radon measures with densities $x^{s} 1_{(0, \infty)}$ on $\mathbb{R}$ with $s>-1$. They converge to the Gaussian exponential famlily if 
$s \rightarrow \infty$ provided we apply a proper normalization. What happens if $s \rightarrow-1$ ? For $s \leq-1$ the measure $x^{s} 1_{(0, \infty)}(x) d x$ is no longer a Radon measure on $\mathbb{R}$. However one can truncate this measure and ask for the limit behaviour of the exponential family of probability measures with densities $f_{\gamma}(x)=c(s, \gamma) e^{\gamma x} x^{s} 1_{[1, \infty)}(x)$, as $\gamma \uparrow \gamma_{\infty}=0$. For $s<-1$ the answer is simple: $\gamma_{\infty}$ lies in $\Gamma$ and Corollary 2.2 applies. If $s=-1$ the situation is more delicate. There exists a non-degenerate limit distribution, but only under non-linear normalization. See Example 4.10.

Weak convergence in (1.3) implies convergence of the mgf's. The significance of this result will become apparant in the next section.

Theorem 3.4 Let (1.3) hold. Suppose $Y$ is non-constant and $\lambda_{\infty} \notin \Lambda$. Then the mgf's of the normalized variables $A_{\lambda} X_{\lambda}$ converge to the mgf of the limit variable $Y$ on the interval $\Gamma=\left\{\gamma: E e^{\gamma Y}<\infty\right\}$

Proof Let $\gamma \in \Gamma$. There exists a unique positive affine transformation $C=C(\gamma)$ so that $C Y \stackrel{\mathrm{d}}{=} Y_{\gamma}$ by Proposition 3.2.

Let $\mu_{n} \uparrow \lambda_{\infty}$ and set $Y_{n}=A_{\mu_{n}} X_{\mu_{n}}$. We write $A_{\lambda} x=\left(x-b_{\lambda}\right) / a_{\lambda}$ and assume that $a_{\lambda}>0$ and $b_{\lambda}$ depend continuously on $\lambda$. We claim that there is a sequence $\lambda_{n} \rightarrow \lambda_{\infty}$ so that $\gamma_{n}=\left(\lambda_{n}-\mu_{n}\right) a_{\mu_{n}} \rightarrow \gamma$ and $C_{n}=A_{\mu_{n}} A_{\lambda_{n}}^{-1} \rightarrow C$.

First assume $\gamma<0$. Set $r:=\sup \{\| C(\xi) \mid \gamma \leq \xi \leq 0\}$. Then $\left\|A_{\mu_{n}}\right\| \rightarrow \infty$ by Proposition 2.10 and hence $\left\|A_{\mu_{n}} A_{0}^{-1}\right\|>r+1$ and $\mu_{n}>0$ for $n \geq n_{0}$. Let $n \geq n_{0}$. Let $\lambda$ decrease from $\mu_{n}$ to 0 . By continuity there is a maximal value $\lambda_{n}$ for which $\gamma_{n}:=$ $\left(\lambda_{n}-\mu_{n}\right) a_{\mu_{n}}=\gamma$ or $\left\|C_{n}:=\right\| A_{\mu_{n}} A_{\lambda_{n}}^{-1} \|=r+1$. (In the latter case $\gamma_{n} \in[0, \gamma]$.) Note that $\lambda_{n} \rightarrow \lambda_{\infty}$ since $\lambda_{k_{n}} \rightarrow \lambda \in\left[0, \lambda_{\infty}\right)$ implies that $\left\|C_{n}\right\| \rightarrow \infty$ by Proposition 2.10. Now assume $\gamma_{n} \rightarrow \beta$ and $C_{n} \rightarrow B$. (Take subsequences if need be.) Then $E_{\gamma_{n}} Y_{n} \stackrel{\mathrm{d}}{=} C_{n} Y^{\lambda_{n}} \stackrel{\mathrm{d}}{\rightarrow}$ $B Y$ by (2.7). Hence $B Y \stackrel{\mathrm{d}}{=} E_{\beta} Y$ and $E e^{\gamma_{n} Y_{n}} \rightarrow E e^{\beta Y}$ by Proposition 2.4. So $B=C(\beta)$, and $\beta \in[\gamma, 0]$ implies $\|B\| \leq r$. Hence eventually $\left\|C_{n}\right\|<r+1$ which implies $\gamma_{n}=\gamma$. Thus we see that $\gamma_{n} \rightarrow \gamma$. The proof for $\gamma \geq 0$ is similar.

Corollary 3.5 If (1.3) holds, convergence to a nondegenerate limit still takes place if $F_{\lambda}$ is centered and scaled by expectation and standard deviation. 


\section{Sequential limits}

In this section we only assume that the limit relation (1.3) holds for a sequence $\lambda_{n} \uparrow \lambda_{\infty}$. As above $\lambda_{\infty}=\sup \Lambda \notin \Lambda$. We adapt the notation slightly. $V$ is a non-degenerate random variable such that

$$
V_{n}:=A_{n} X_{\lambda_{n}}=\left(X_{\lambda_{n}}-b_{n}\right) / a_{n} \stackrel{\mathrm{d}}{\rightarrow} V .
$$

We treat two questions. 1) What information does the sequence $\left(A_{n}\right)$ give about the distribution of the limit variable $V$ ? 2) What limit distributions are possible in (4.1)?

Proposition 4.1 Suppose (4.1) holds. If $A_{n+1} A_{n}^{-1} \rightarrow$ id then (1.3) holds: There exist functions $a(\lambda)>0$ and $b(\lambda)$ so that $\left(X_{\lambda}-b(\lambda)\right) / a(\lambda) \stackrel{\mathrm{d}}{\rightarrow} V$.

Proof Khintchine's convergence of types theorem implies $A_{n} X_{\lambda_{n+1}} \stackrel{\mathrm{d}}{\rightarrow} V$. Set $A(\lambda)=A_{n}$ for $\lambda_{n} \leq \lambda<\lambda_{n+1}$. Monotonicity of $\lambda \mapsto F_{\lambda}(x)$, see Proposition 2.1, ensures that $A(\lambda) X_{\lambda} \stackrel{\mathrm{d}}{\rightarrow} V$ for $\lambda \rightarrow \lambda_{\infty}$.

Now assume $A_{n+1} A_{n}^{-1} \rightarrow C \neq \mathrm{id}$. In the asymptotic theory of sums or maxima this implies that the limit, if it exists, is semistable. See Hazod \& Scheffler [1993]. For exponential families semistability means that the limit distribution belongs to $\mathcal{F}(\gamma, C)$ for some $\gamma \neq 0$. However the situation for exponential families is more complex than for sums or maxima. We shall investigate the behaviour for translations.

Example 4.2 An integer valued limit variable.

Construction Let the random integer $X$ have a logconcave distribution with $P\{X=k\}$ $=p_{k}=e^{-\alpha_{k}}>0$ for all $k$. So $p_{k-1} p_{k+1} \leq p_{k}^{2}$. Assume $p_{k+1} p_{k-1} / p_{k}^{2} \rightarrow e^{-\gamma} \in(0,1)$ for $k \rightarrow \infty$. Let $\lambda_{k}=\left(\alpha_{k-1}+\alpha_{k+1}\right) / 2$. Then $X_{\lambda_{n}}-n \stackrel{\mathrm{d}}{\rightarrow} V$ with $V$ as defined in (3.1).

Now suppose $\left(\lambda_{n}^{\prime}-\lambda_{n}\right) / \gamma \rightarrow \alpha \in \mathbb{R}$. Then $X_{\lambda_{n}^{\prime}}-n \stackrel{\mathrm{d}}{\rightarrow} V^{\prime}$ where $P\left\{V^{\prime}=k\right\}=$ $e^{-\gamma(k-\alpha)^{2} / 2} / c(\alpha)$. All limit variables $V^{\prime}$ belong to $\mathcal{F}(\gamma, C)$ where $C$ is the translation $C x=x+1$.

The exponential family $F_{\lambda}$ of the $\operatorname{rv} X$ in the example above gives rise to a oneparameter exponential family of limit distributions $G_{\lambda}, \lambda \in \mathbb{R}$. We are only interested in limit types. Since $G_{\lambda+1 / 2 \gamma}(x)=G_{\lambda}(x-1)$ the limit types in this example form a compact family. Topologically this family is a circle. Let $[F]$ denote the type of the df $F$. As in the case of semi-stable limit distributions for sums and maxima one may describe the 
behaviour of the family of types $\left[F_{\lambda}\right], \lambda \in \Lambda$, for $\lambda \rightarrow \lambda_{\infty}$ as a curve which spirals to a limiting circle in the space of distribution types.

For exponential families there is an additional limit family. This limit family has no counterpart in the asymptotic theory of sums or maxima.

Choose the weights $p_{k}$ above so that $p_{k+1} p_{k-1} / p_{k}^{2} \rightarrow 0$. The possible non-constant limit distributions of the sequence $X_{\mu_{n}}-n$ then are members of the exponential family of Bernoulli variables, $P\left\{V_{\lambda}=1\right\}=1 /\left(1+e^{-\lambda}\right)=1-P\left\{V_{\lambda}=0\right\}$, together with the constant variable $V \equiv 0$.

We shall now adapt this example so as to obtain a compact "circle" of non-degenerate limit types.

Example 4.3 A rv X so that the set of limit types of the exponential family is a "circle" consisting of the types of the following rv's: $U_{\gamma}, \gamma \in \mathbb{R}, E, W_{\gamma},-1<\gamma<1,-E$. Here $E$ is exponentially distributed, $U_{\gamma}$ is the exponential family generated by the uniform $(0,1) \mathrm{rv}$ $U$ and $W_{\gamma}$ is the exponential family generated by the $r v W$ with Laplace density $e^{-|x|} / 2$.

Construction Let $X$ have density $f=e^{-\varphi}$ where $\varphi: \mathbb{R} \rightarrow \mathbb{R}$ is a convex function which is piecewise linear on each interval $[k, k+1]$ with slope $\lambda_{k}$. Assume that $\lambda_{k+1}-\lambda_{k} \rightarrow \infty$ for $k \rightarrow \infty$. Then $X_{\lambda_{n}}-n \stackrel{\mathrm{d}}{\rightarrow} U$ where $U$ is uniformly distributed on the interval $[0,1]$. The reader can easily check that the set of limit types has the given form.

The rv $X$ with density $f=e^{\varphi}$ in the example above has the following property: There is a continuous family of non-degenerate limit distributions $G^{\theta}, 0 \leq \theta \leq 2 \pi$, all of different type, except that $G^{0}=G^{2 \pi}$, a continuous curve $A: \Lambda \rightarrow \mathcal{G}$ and a continuous strictly increasing function $\psi: \Lambda \rightarrow \mathbb{R}$ tending to infinity for $\lambda \rightarrow \lambda_{\infty}$ so that for each $\theta \in[0,2 \pi]$

$$
A\left(\lambda_{n}\right) X_{\lambda_{n}} \stackrel{\mathrm{d}}{\rightarrow} V^{\theta} \sim G^{\theta}
$$

whenever $\lambda_{n} \uparrow \lambda_{\infty}$ and $e^{i \psi\left(\lambda_{n}\right)} \rightarrow e^{i \theta}$.

In particular the set of df's $F_{\lambda}, \lambda \in \Lambda \cap[0, \infty)$ is stochastically compact: Any sequence $F_{\lambda_{n}}$ contains a subsequence which may be normed to converge weakly to a non-degenerate limit distribution. See de Haan \& Resnick [1984]. The family of all possible limit variables $a V^{\theta}+b$ is closed under the Esscher transform. It contains rv's from the extended gamma family but also bounded rv's and unbounded rv's which are not semi-stable. 
These two examples give an indication of the behaviour of the sequence $X_{\lambda_{n}}$ under the condition that $A_{n} A_{n+1}^{-1} \rightarrow C \neq$ id. However in order that the limit distribution in (4.1) belong to the class $\mathcal{F}(\gamma, C)$ it is not necessary that the sequence $A_{n} A_{n+1}^{-1}$ converge. Large gaps may occur. From Section 2 we know that the limit $V$ belongs to $\mathcal{F}(\gamma, C)$ if there exist integer sequences $q_{n} \rightarrow \infty$ and $k_{n}>q_{n}$ so that $A_{q_{n}} A_{k_{n}}^{-1} \rightarrow C$ and $\left(\lambda_{k_{n}}-\lambda_{q_{n}}\right) a_{q_{n}} \rightarrow \gamma$. We therefore introduce the set $\mathcal{H}_{0}$ of all $C \in \mathcal{G}, C \neq \mathrm{id}$, which are limit of a sequence $C_{n}=A_{q_{n}} A_{k_{n}}^{-1}$ with $k_{n}>q_{n} \rightarrow \infty$.

Relation (2.7) gives

$$
C_{n} V_{k_{n}} \stackrel{\mathrm{d}}{=} E_{\gamma_{n}} V_{q_{n}}
$$

where we write $C_{n}=A_{q_{n}} A_{k_{n}}^{-1}$ as above and $\gamma_{n}=\left(\lambda_{k_{n}}-\lambda_{q_{n}}\right) a_{q_{n}}$. Since we assume that $C_{n} \rightarrow C$ Theorem 2.7 applies: $\gamma_{n} \rightarrow \gamma \in[0, \infty]$. If $\gamma=\infty$ there exists a constant $c$ such that $V \leq c \leq C V$ and the $\mathrm{rv} V$ is bounded. If $\gamma$ is finite then $C V \stackrel{\mathrm{d}}{=} V_{\gamma}$ and $V$ belongs to the set $\mathcal{F}(\gamma, C)$.

This yields the following dichotomy:

Theorem 4.4 If $\mathcal{H}_{0}$ is non-empty then either

$V$ is bounded and $\gamma=\infty$ for each $C \in \mathcal{H}_{0}$, or

$V$ is unbounded and $\gamma$ is finite for each $C \in \mathcal{H}_{0}$.

Our next result extends Proposition 4.1 and is a partial converse to Theorem 3.3.

Theorem 4.5 Suppose (4.1) holds and the sequence $\left(\left\|A_{n} A_{n+1}^{-1}\right\|\right)$ is bounded. If the limit variable belongs to the extended gamma family then (1.3) holds.

Proof The limit variable $V$ satisfies the stability relations $C^{t} V \stackrel{\mathrm{d}}{=} E_{\gamma(t)} V, t \in \mathbb{R}$, see Proposition 3.2. Write $\left(\lambda_{n+1}-\lambda_{n}\right) a_{n}=\gamma_{n}=\gamma\left(t_{n}\right)$. The sequence $\left(t_{n}\right)$ is bounded. Equivalent are: $t_{k_{n}} \rightarrow t_{0}, \gamma_{k_{n}} \rightarrow \gamma_{0}=\gamma\left(t_{0}\right)$ and $A_{k_{n}} A_{k_{n}+1}^{-1} \rightarrow C^{-t_{0}}$. These relations imply $E_{\gamma_{k_{n}}} V_{k_{n}}=A_{k_{n}+1} A_{k_{n}}^{-1} V_{k_{n}} \stackrel{\mathrm{d}}{\rightarrow} C^{t_{0}} V=E_{\gamma_{0}} V$ and (by Proposition 2.4) $E e^{\gamma_{k_{n}} V_{k_{n}}} \rightarrow E e^{\gamma_{0} V}$.

Now define

$$
A(\lambda):=C^{-s} A_{n} \quad \lambda=\lambda_{n}+\gamma(s) / a_{n}, \quad 0 \leq s<t_{n} .
$$

We have to prove that $A\left(\mu_{n}\right) X_{\mu_{n}} \stackrel{\mathrm{d}}{\rightarrow} V$ for any sequence $\mu_{n} \uparrow \lambda_{\infty}$. It suffices to consider sequences $\mu_{n}=\lambda_{j_{n}}+\alpha_{n} / a_{j_{n}}$ with $\alpha_{n}=\gamma\left(s_{n}\right) \rightarrow \alpha_{0}=\gamma\left(s_{0}\right)$ for $0 \leq s_{n}<t_{j_{n}}$. Then

$$
A\left(\mu_{n}\right) X_{\mu_{n}} \stackrel{\mathrm{d}}{=} C^{-s_{n}} A_{j_{n}} E_{\alpha_{n} / a_{j_{n}}} X_{\lambda_{j_{n}}}=C^{-s_{n}} E_{\alpha_{n}} V_{j_{n}}
$$


Now observe $s_{n} \rightarrow s_{0}, \alpha_{n} \rightarrow \alpha_{0}$. The bound $0 \leq \alpha_{n} \leq \gamma_{n}$ implies $E e^{\alpha_{n} V_{j_{n}}} \rightarrow E e^{\alpha_{0} V}$ and hence $E_{\alpha_{n}} V_{j_{n}} \stackrel{\mathrm{d}}{\rightarrow} E_{\alpha_{0}} V$ and $A\left(\mu_{n}\right) X_{\mu_{n}} \stackrel{\mathrm{d}}{\rightarrow} C^{-s} E_{\gamma(s)} V \stackrel{\mathrm{d}}{=} V$.

The condition that the limit variable belong to the extended gamma family is less restrictive than it seems. Since the sequence $A_{n} A_{n+1}^{-1}$ is bounded, the set $\mathcal{H}_{0}$ is nonempty. Hence the condition will be satisfied if 1) $\mathcal{H}_{0}$ contains a sequence $C_{n} \rightarrow$ id, or 2) $V$ is unbounded and $\mathcal{H}_{0}$ is not contained in a discrete subgroup $C^{k}, k \in \mathbb{Z}$, of $\mathcal{G}$.

Without conditions on the sequence $A_{n}$ every limit law is possible in (4.1).

Example 4.6 A Cauchy-distributed limit variable $V$ is possible in (4.1).

Proof We shall construct a rv $X$ with density $f$ so that $X_{\lambda_{n}}-\lambda_{n}$ converges to a rv $V$ with density $1 / \pi\left(1+x^{2}\right)$ for $\lambda_{n}=n^{2}$.

Let $I_{n}$ be the interval $[-\sqrt{n}, \sqrt{n}]$ and define

$$
h_{n}(u)=\frac{e^{u^{2} / 2}}{\pi\left(1+u^{2}\right)} 1_{I_{n}}(u) \quad n \geq 1 .
$$

Now introduce $h$ as maximum of translates of the functions $h_{n}$ : Set $h\left(n^{2}+u\right)=h_{n}(u)$ for $n \geq 1$ and $u \in I_{n}$ and set $h(x)=0$ elsewhere. Similarly define $h^{*}\left(n^{2}+u\right)=e^{n}$ for $n \geq 1$ and $u \in I_{n}$ and $h^{*}(x)=0$ elsewhere. Then $h \leq h^{*}$. Define $g(x):=h(x) e^{-x^{2} / 2}$ and $g_{\lambda}(x):=g(x) e^{\lambda x} / e^{\lambda^{2} / 2}$. A simple computation gives

$$
g_{n}^{*}(u):=g_{\lambda_{n}}\left(n^{2}+u\right)=h\left(n^{2}+u\right) e^{-u^{2} / 2} .
$$

this means that $g_{n}^{*}(u)=1 / \pi\left(1+u^{2}\right)$ on $I_{n}$ and $g_{n}^{*}(u)=0$ for $\sqrt{n}<|u| \leq n$. The tails of $g_{n}^{*}$ are negligible: Lemma 4.7 below implies that

$$
g_{n}^{*}(u) \leq h^{*}\left(n^{2}+u\right) e^{-u^{2} / 2} \leq e^{-u^{2} / 6} \quad n \geq 4,|u| \geq n
$$

Hence $\left\|g_{n}^{*}\right\|_{1} \rightarrow 1$. Now let the $\mathrm{rv} X$ have density $f=g / c$ with $c=\|g\|_{1}$. Then $X_{\lambda_{n}}-n^{2}$ has density $g_{n}^{*} / c_{n}$ for $n \geq 1$ where $c_{n}=\left\|g_{n}^{*}\right\|_{1} \rightarrow 1$.

Lemma 4.7 The function $h^{*}$ in Example 4.6 satisfies the inequality

$$
h^{*}\left(n^{2}+u\right) \leq e^{u^{2} / 3} \quad|u| \geq n, n \geq 4 .
$$

Proof Introduce the concave piecewise linear function $\psi:[0, \infty) \rightarrow[0, \infty)$ with the value $n$ in $n^{2}-n$ for $n \geq 1$. Then $h^{*} \leq e^{\psi}$ and $\psi\left(m^{2}+u\right) \leq u^{2} / 3$ for $|u| \geq m$ and $m \geq 4$. (The inequality holds in $u= \pm m$ and $\psi^{\prime}\left(m^{2}+u\right)=1 /(2 m+1) \leq 2 u / 3$ in $u=m+0$.) 
Doeblin introduced the concept of universal distributions in his study of the asymptotic behaviour of sums of iid rv's. Let $S_{n}$ be the sum of the first $n$ terms of a sequence of independent samples from the df $F$. The distribution $F$ is universal if for each rv $V$ there exists a subsequence $k_{1}<k_{2}<\cdots$ and a sequence of positive affine normalizations $A_{n}$ so that $A_{n} S_{k_{n}} \stackrel{\text { d }}{\rightarrow} V$. Doeblin [1946] established the existence of universal distributions. See Feller [1966, vol. 2, p. 555] for details. One can introduce a similar concept for exponential families. An exponential family $X_{\lambda}, \lambda \in \Lambda$, is universal if for each rv $V$ there exists a sequence $\lambda_{n} \uparrow \lambda_{\infty}=\sup \Lambda$ and a sequence of positive affine transformations $A_{n}$ so that $A_{n} X_{\lambda_{n}} \stackrel{\text { d }}{\rightarrow} V$.

Theorem 4.8 Universal exponential families exist.

Proof First note that there exists a sequence of df's $Q_{n}$ on $\mathbb{R}$ which is dense in the space of all probability distributions with the topology of weak convergence. We can choose the df's $Q_{n}$ to have a continuous density $q_{n}$ which is bounded by $e^{n / 2}$ and which vanishes outside the interval $I_{n}=[-\sqrt{n}, \sqrt{n}$. The construction of Example 4.6 yields a rv $X$ with density $f$ such that $X_{\lambda_{n}}-\lambda_{n}$ has density $g_{n} / c_{n}$ where $g_{n}$ agrees with $q_{n}$ on $[-n, n]$ and $c_{n} \rightarrow 1$ since the function $g_{n}^{*}$ is bounded by $e^{-x^{2} / 6}$ outside the interval $[-n, n]$ for $n \geq 4$. As in the example we take $\lambda_{n}=n^{2}$.

Let $V$ be a rv with df $Q$. There is a sequence $k_{n} \uparrow \infty$ so that $Q_{k_{n}} \rightarrow Q$ weakly. Then $X_{\mu_{n}}-\mu_{n} \stackrel{\text { d }}{\rightarrow} V$ if we choose $\mu_{n}=k_{n}^{2}$.

Universal exponential families have the property that any $\mathrm{df} Q(x)$ is limit of some sequence $F_{\lambda_{n}}\left(a_{n} x+b_{n}\right)$ with $\lambda_{n} \uparrow \lambda_{\infty}$. With more effort one can show that this is also possible under the additional restriction that the sequences $\lambda_{n}$ are asymptotically dense: $\lambda_{n+1}-\lambda_{n} \rightarrow 0$. This result will be published elsewhere.

A further question of interest is: Do there exist non-degenerate df's $F$ such that only degenerate limit distributions are possible in (4.1)?

If the df $F$ of $X$ has a jump in its upper endpoint $x_{\infty}$ then $P\left\{X_{\lambda}=x_{\infty}\right\} \rightarrow 1$ and only a degenerate limit is possible in (4.1). Less trivial examples are:

Example 4.9 Let $F$ have density $f(x)=c e^{-x} / x$ on $x \geq 1$. Then (4.1) will hold only for constant limit variables $V$.

Proof $Z_{\epsilon}=\epsilon X_{1-\epsilon}$ has density $f_{\epsilon}(x)=c(\epsilon) e^{-x} / x$ on $[\epsilon, \infty)$. If $\epsilon \downarrow 0$ then $f_{\epsilon}(x) / c(\epsilon) \rightarrow$ $e^{-x} / x$ on $(0, \infty)$ and $c(\epsilon) \sim \log (1 / \epsilon)$. Hence $F_{\epsilon}\left(\epsilon^{u}\right) \rightarrow 1-u$ for $u \in(0,1)$ for $\epsilon \downarrow 0$. Take 
$0<u<<1$. The half line $\left[\epsilon^{u}, \infty\right)$ carries weight $u>0$, but a large part, $1-2 u$, of the probability lives on the relatively short interval $\left[0, \epsilon^{2 u}\right]$. Since $\epsilon^{2 u}=o\left(\epsilon^{u}\right)$ there is an atom of weight $\geq 1-2 u$ in the limit. Because $u>0$ is arbitrary the limit can only be degenerate.

Note that $F_{\epsilon}\left(\epsilon^{u}\right) \rightarrow 1-u$ implies that $\log \left(X_{1-\epsilon}\right) / \log (1 / \epsilon) \stackrel{\text { d }}{\rightarrow} U$ where $U$ is uniformly distributed on $(0,1)$. This means that the exponential family $X_{\lambda}$ has a non-degenerate limit under power norming: Take $c(\lambda)=-\log (1-\lambda)$ then

$$
X_{\lambda}^{1 / c(\lambda)} \stackrel{\mathrm{d}}{\rightarrow} e^{U} \quad \lambda \rightarrow 1
$$

For recent work on power norming for extremes, see Pancheva [1984] and Ravi [1991]. With the arguments of Example 4.9 one may show:

Example 4.10 Let $F$ have density $f(x)=c e^{-x}(\log x)^{\alpha} / x$ on $(e, \infty)$. For $\alpha \geq-1$ the limit relation (4.1) has only constant limits. Let $U$ be uniformly distributed on $[0,1]$. If $\alpha>-1$ then

$$
\frac{\log X_{\lambda}}{\log (1 /(1-\lambda))} \stackrel{\mathrm{d}}{\rightarrow} U^{1 /(1+\alpha)} \quad \lambda \rightarrow \lambda_{\infty}=1 .
$$

If $\alpha=-1$ then even power norming yields only constant limit variables but

$$
\left(\log \log X_{\lambda}\right) / \log \log (1 /(1-\lambda)) \stackrel{\mathrm{d}}{\rightarrow} U, \quad \lambda \rightarrow 1 .
$$

Let $[F]$ denote the type of the non-degenerate $\mathrm{df} F$. In this paper we have studied the behaviour of the curve $\left[F_{\lambda}\right]$ in the space of non-degenerate probability types. Introduce $\Xi(F)$ as the set of limits of sequences $\left[F_{\lambda_{n}}\right]$ with $\lambda_{n} \uparrow \lambda_{\infty}$. If $\left[F_{\lambda}\right]$ converges to a point $[G]$ then $\Xi(F)$ is a singleton and $[G]$ belongs to the one-parameter extended gamma type family of Example 2.9. We have seen examples where the limit set $\Xi(F)$ is a circle, a line, the whole space of types (for universal exponential families) and the empty set (Examples 4.9 and 4.10).

The asymptotic behaviour of the tail of the $\mathrm{df} F$ is reflected in the asymptotic behaviour of the tail of the mgf. The exponential family $F_{\lambda}$ for $\lambda \uparrow \lambda_{\infty}$ describes the tail behaviour of $F$. In terms of the $\operatorname{cgf} \kappa$ the exponential family consists of translates of the graph of this convex function $\kappa$. It is not clear how the wide range of behaviour of the curve $\left[F_{\lambda}\right]$ hinted at in this section is reflected in the asymptotic behaviour of the convex analytic function $\kappa$. More insight in this question should lead to a better understanding of the relation between the tail behaviour of a df and its mgf. 
Acknowledgments. Research on this topic was started during a visit to Cornell University some years ago by A.A. Balkema and C. Klüppelberg. They would like to thank the Department of Operations Research and Industrial Engineering for its hospitality. We would also like to thank one of the referees for the very careful reading of our paper and the detailed comments.

\section{References}

Balkema, A.A., Klüppelberg, C. and Resnick, S.I. (1993). Densities with Gaussian tails. Proc. London Math. Soc. (3) 66 568-588.

Balkema, A.A., Klüppelberg, C. and Stadtmüller, U. (1995). Tauberian results for densities with Gaussian tails. J. London Math. Soc. (2) 51 383-400.

Bar-Lev, S.K. and Casalis, M. (1994). Les familles exponentielles naturelles reproduisantes C.R. Acad. Sci. Paris 319 1323-1326.

Bar-Lev, S.K. and Casalis, M. (1998) Reproducibility of the real natural exponential families. Submitted to Probab. Th. Rel. Fields.

Barndorff-Nielsen, O.E. (1978). Information and Exponential Families in Statistical Theory. Wiley, Chichester.

Barndorff-Nielsen, O.E. and Cox, D.R. (1994). Inference and Asymptotics. Chapman and Hall, London.

Barndorff-Nielsen, O.E. and Klüppelberg, C. (1997). Tail exactness of multivariate saddlepoint approximations. Scand. Statist. J. To appear.

Broniatowski, M. and Mason, D. (1994). Extended large deviations. J. Theor. Prob. 7 647-666.

Brown, L. (1986). Fundamentals of statistical exponential families. Institute of Math. Stat. Lecture notes - Monograph series, Vol. 9; ed. S. Gupta. Hayward.

Casalis, M. (1991) Familles Exponentielles Naturelles sur $\mathbb{R}^{d}$ Invariantes par une Groupe. Internat. Statist. Review59 241-162. 
Davis, R. and Resnick, S.I. (1991). Extremes of moving averages of rv's with finite endpoint. Ann. Probab. 19 312-328.

Doeblin, W. (1946). Sur l'ensemble de puissances d'une loi de probabilité Ann. Ec. Norm. Sup. (III) 63 317-350.

Feigin, P. and Yashchin, E. (1983). On a strong Tauberian result. Z. Wahrsch. verw. Gebiete 65 35-48.

Feller, W. (1969). Limit theorems for probabilities of large deviations. Z. Wahrsch. verw. Gebiete 14 1-20.

Feller, W. (1971). An Introduction to Probability Theory. Vol.2. Wiley, New York.

Haan, L. de (1970) On Regular Variation and Its Application to Weak Convergence of Sample Extremes. CWI Tract 32, Amsterdam.

Haan, L. de and Resnick, S. I. (1984). Asymptotically balanced functions and stochastic compactness of sample extremes. Ann. Probab.12 588-608.

Hazod, W. and Scheffler, H.P. (1993). The domains of partial attraction of probabilities on groups and on vector spaces. J. Theor. Probab.6 175-186.

Jensen, J.L. (1995). Saddlepoint Approximations. Oxford U.P.

Lehmann, E.L. (1983). Theory of Point Estimation. Wiley, New York.

Pancheva, E. (1984). Limit theorems for extreme order statistics under non-linear normalization. Lect. Notes Math. 1155 284-309.

Ravi S. (1991). Tail equivalence of df's belonging to the max domains of attraction of univariate p-max stable laws. J. Indian Stat. Assoc. 29 69-77.

Rootzen, H. (1987). A ratio limit theorem for the tails of weighted sums. Ann. Probab. $15728-747$.

Teicher, H. (1984). The CLT for Esscher transformed rv's. Sankhya A46 35-40. 